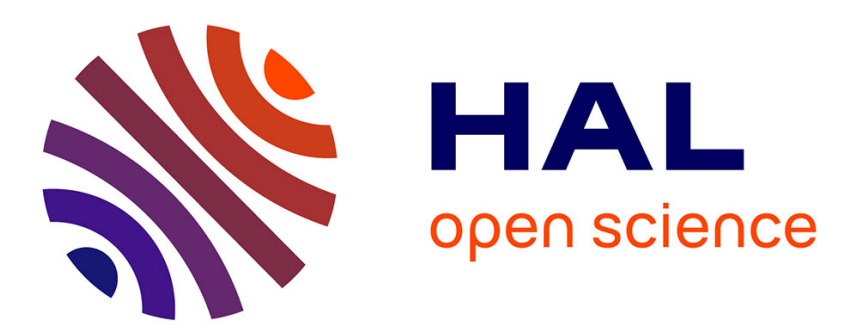

\title{
Ecologie d'un espace géographique. Les géosystèmes du Valle de Prioro (Espagne du Nord-Ouest)
}

Georges Bertrand

\section{To cite this version:}

Georges Bertrand. Ecologie d'un espace géographique. Les géosystèmes du Valle de Prioro (Espagne du Nord-Ouest). Espace Géographique, 1972, 1 (2), pp.113-128. 10.3406/spgeo.1972.1319 . hal02570238

\section{HAL Id: hal-02570238 \\ https://hal.science/hal-02570238}

Submitted on 11 May 2020

HAL is a multi-disciplinary open access archive for the deposit and dissemination of scientific research documents, whether they are published or not. The documents may come from teaching and research institutions in France or abroad, or from public or private research centers.
L'archive ouverte pluridisciplinaire HAL, est destinée au dépôt et à la diffusion de documents scientifiques de niveau recherche, publiés ou non, émanant des établissements d'enseignement et de recherche français ou étrangers, des laboratoires publics ou privés. 


\section{Ecologie d'un espace géographique.}

Les géosystèmes du Valle de Prioro (Espagne du Nord-Ouest)

Georges Bertrand

\section{Citer ce document / Cite this document :}

Bertrand Georges. Ecologie d'un espace géographique.. In: Espace géographique, tome 1, n², 1972. pp. 113-128;

doi : https://doi.org/10.3406/spgeo.1972.1319

https://www.persee.fr/doc/spgeo_0046-2497_1972_num_1_2_1319

Fichier pdf généré le 03/01/2019 


\title{
Résumé
}

Exemple d'application d'une méthode d'étude intégrée des milieux physiques qui combine en les harmonisant les démarches écologique et géographique. Un inventaire des données, sectoriel dans son principe mais strictement orienté vers l'étude des combinaisons et des interactions entre les éléments du milieu, précède l'analyse globale proprement dite qui examine et classe les différentes unités isomorphes : géosystèmes et géofaciès (cf. carte) . Les paysages "physico- anthropiques »du Valle de Prioro, bassin- versant de la moyenne montagne cantabrique, se caractérisent par la fragilité de leurs structures, en particulier au niveau hydrologique et phytogéographique. La dynamique des paysages, régressive dans son ensemble, est très nuancée dans le détail des géofaciès. Elle dépend pour l'essentiel du mode d'utilisation de l'espace de la société montagnarde et plus particulièrement du système pastoral.

\begin{abstract}
An example of the application of an integrated method of study in physical milieus which combines the ecological and geographical steps in their harmonisation. An inventory of the data, by theoretical sectors but strictly directed towards the study of combinations and interactions between the elements of the environment, precedes the properly so called total analysis which examines and classifies the different isomorphic units: geosystems and geofacies (see map). The physical and anthropic landscapes of the Valle de Prioro, a river basin of the intermediate Cantabrian Mountains is characterized by their structural fragility, in particular on the hydrological and hydrographical level. The dynamics of the landscapes, regressive as a whole, is very varied in appearance. It depends essentially on the method of the use of space by the mountain society and more particularly on the pastoral system.
\end{abstract}




\title{
Ecologie d'un espace géographique : LES GÉOSYSTÈMES DU VALLE DE PRIORO (Espagne du Nord-Ouest)
}

\author{
Georges BERTRAND \\ C.I.M.A. (Centre Interdisciplinaire de recherches \\ sur les Milieux physiques et leur Aménagement), \\ Institut de Géographie, Université de Toulouse - Le Mirail
}

\begin{abstract}
RESUME. Exemple d'application d'une méthode d'étude intégrée des milieux physiques qui combine en les harmonisant les démarches écologique et géographique. Un inventaire des données, sectoriel dans son principe mais strictement orienté vers l'étude des combinaisons et des interactions entre les éléments du milieu, précède l'analyse globale proprement dite qui examine et classe les différentes unités isomorphes: géosystèmes et géofaciès (cf. carte). Les paysages «physicoanthropiques» du Valle de Prioro, bassin-versant de la moyenne montagne cantabrique, se caractérisent par la fragilité de leurs structures, en particulier au niveau hydrologique et phytogéographique. La dynamique des paysages, régressive dans son ensemble, est très nuancée dans le détail des géofaciès. Elle dépend pour l'essentiel du mode d'utilisation de l'espace de la société montagnarde et plus particulièrement du système pastoral.
\end{abstract}

ABSTRACT. An example of the application of an integrated method of study in physical milieus which combines the ecological and geographical steps in their harmonisation. An inventory of the data, by theoretical sectors but strictly directed towards the study of combinations and interactions between the elements of the environment, precedes the properly so called total analysis which examines and classifies the different isomorphic units: geosystems and geofacies (see map). The physical and anthropic landscapes of the Valle de Prioro, a river basin of the intermediate Cantabrian Mountains is characterized by their structural fragility, in particular on the hydrological and hydrographical level. The dynamics of the landscapes, regressive as a whole, is very varied in appearance. It depends essentially on the method of the use of space by the mountain society and more particularly on the pastoral system.

Par ses paysages horizuntaux, décharnés et poussiéreux, le Valle de Prioro introduit en pleine montagne cantabrique des aspects de páramo castillan (1). C'est l'un des nombreux alvéoles de la grande dépression intramontagneuse qui à̀re le versant sud du massif cantabrique entre les méridiens de León et Palencia, à mi-distance entre les hauts reliefs de la divisoria (1) et les plateaux caillouteux du piémont de Vieille Castille (fig. 1). Le Valle de Prioro correspond à la partie amont du bassin versant du rio Cea, affluent du rio Esla, sous-affluent du Duero. Dès l'abord, deux paysages s'individualisent:

Le Haut Prioro, entre 1300 et $1800 \mathrm{~m}$ d'altitude, forme une ceinture presque continue de massifs aux pentes raides et lacérées par l'érosion torrentielle.

(1) Páramo: plateau du piémont castillan; ligne de partage des eaux, ici entre le versant cantabrique et le versant castillan. Divisoria: ligne de partage des eaux.
La mosaïque des paysages y est vigoureusement contrastée : dans les secteurs élevés, des versants structuraux dénudés avec des sols et des manteaux de débris disposés en guirlandes ou accumulés dans les vallons secondaires; sur les ombrées, des massifs forestiers (Hêtraie-Chênaie) stables et homogènes; des formations arborées claires sur des pavages schisteux mobiles; quelques champs de lapiés colonisés par de maigres garides à Thym (2).

Le Bas Prioro, entre 1100 et $1300 \mathrm{~m}$ d'altitude, étale ses paysages monotones et dégradés sur les replats étagés et dans les vallées alluviales qui occupent le centre de la cuvette : des plateaux d'interfluves presque entièrement couverts par le fin damier de l'openfield; sur les collines, des taillis de Chênes embroussaillés; des landes ouvertes sur des pavages

(2) Garide : garrigue de montagne. 


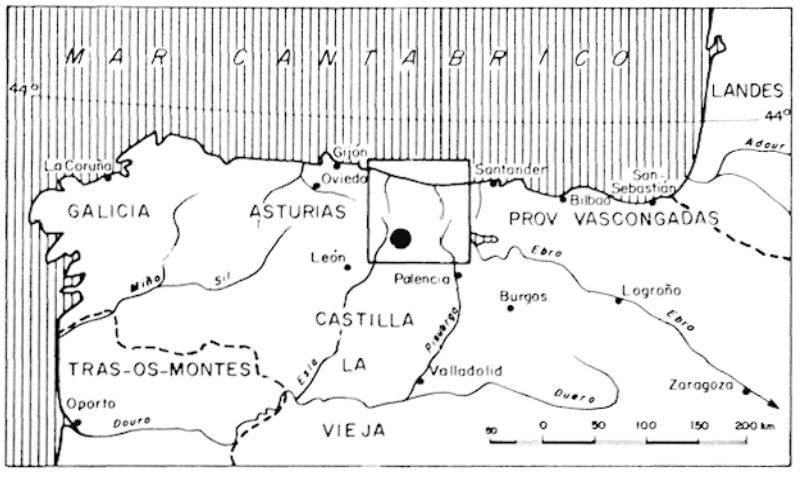

Fra. 1. - Croquis de localisation.

Le point noir représente le Valle de Prioro, le rectangle blanc délimite le secteur des montagnes cantabriques centrales qui a été étudié et cartographié selon la méthode géographique globale.

instables; un réseau envahissant de ravines et de roubines autour des villages et le long des cañadas (3).

Deux impressions, apparemment contradictoires, se dégagent de cette analyse préalable : l'empreinte généralisée d'une "sécheresse » aussi marquée dans la composition floristique et la structure du couvert végétal que dans les modelés actuels des versants; l'existence latente d'une certaine "humidité » qui ne se manifeste que dans quelques aspects particuliers de la végétation et des sols, mais qui s'affirme dans les traits du climat. En somme, des paysages «secs》 sous un climat «humide». Cette remarque ne relève pas du paradoxe. Elle souligne le profond déséquilibre écologique de l'ensemble du Valle de Prioro.

La méthode globale que l'on se propose de suivre n'est pas synthétique dans tous ses développements [3] (4). Ce serait confondre la finalité de la recherche avec la démarche méthodologique. L'étude intégrée des paysages doit presque obligatoirement être précédée par un inventaire analytique des données. Ce préalable est d'autant plus indispensable que les montagnes cantabriques centrales sont assez mal connues sur le plan géographique.

\section{L'INVENTAIRE DES DONNÉES}

\section{Un amphithéâtre montagneux face au sud.}

Le Valle de Prioro est un bassin intramontagneux (1 100-1800 $\mathrm{m}$ d'altitude), ramassé $(7-12 \mathrm{~km} \mathrm{W-E,}$ 6-7 km N-S, soit $49 \mathrm{~km}^{2}$ ), bien circonscrit (le Cea franchit une gorge vers l'aval) et dissymétrique. Le système de pentes est grossièrement concentrique. Les parois rocheuses et les longs versants raides (pentes moyennes supérieures à $40 \%$ ) forment un

(3) Cañada (espagnol) : draille, voie de transhumance, chemin pastoral.

(4) G. BERTRand [3]. Les numéros en grasses renvoient à la bibliographie. rebord presque continu, renforcé par des massifs aux corniches subverticales. En contre-bas, se développe une auréole de pentes moyennes mais irrégulières de l'ordre de 15 à $35 \%$. Le centre de la cuvette, zone de convergence hydrographique, est occupé par des pentes faibles ou nulles, inférieures à $15 \%$. Ce système de versants en amphithéâtre tourné vers le Sud joue un rôle essentiel dans la distribution et l'écologie des milieux géographiques. Il résulte d'un évidement, rythmé par un emboîtement simple de topographies morphoclimatiques, dans une trame structurale particulièrement tourmentée. Les montagnes cantabriques centrales appartiennent en effet à une zone externe non granitisée et très différenciée d'un massif ancien réincorporé et remodelé dans un orogène tertiaire. Le Valle de Prioro correspond à une vaste déformation synclinoriale qui affecte les séries schisteuses carbonifères (5).

Les crêtes structurales qui ceinturent le bassin représentent les racines des synclinaux hercyniens (calcaires cristallins, grès quartzites, conglomérats calcaires ou quartzitiques). Les déformations tectoniques, vigoureuses et embrouillées, ne donnent cependant que des formes structurales simples et presque monotones du fait de la vigueur uniforme des pendages (45-90") : barres, hog-backs, crêts normaux ou de flanc inverse (synclinal de Tejerina). L'altitude de ces crêtes $(1550-1800 \mathrm{~m})$ et la raideur des pentes $(50-80 \%)$ sont deux facteurs écologiques limitants très uniformes. Par contre, les lithofaciès calcaires et les lithofaciès gréseux ou quartzitiques donnent des substrats à la fois très différenciés et très imbriqués. Le massif de Cueto, au SW du Valle de Prioro, porte sur sa face nord, entre $1718 \mathrm{~m}$ et $1600 \mathrm{~m}$, l'empreinte d'un cirque glaciaire isolé que bordent vers l'aval les bourrelets morainiques du Maximum.

Les replats étagés qui tronquent les schistes tendres (faciès Culm) du centre-sud du bassin correspondent à l'enracinement des rañas du piémont castillan à l'intérieur de la montagne cantabrique (6). Il y a au moins deux générations de formes emboîtées: d'une part, des aplanissements sub-horizontaux entre 1250 et $1350 \mathrm{~m}$ d'altitude que voilent localement les cailloutis patinés des rañas; d'autre part, des surfaces en pente douce entre $1150 \mathrm{~m}$ et $1180 \mathrm{~m}$ d'altitude qui convergent vers la basse gouttière du Cea. Ce système de replats étagés, assimilables à des glacis d'érosion en roche tendre, a été élaboré au cours du Pliocène et du Villafranchien. Il caractérise la phase finale du grand évidement synorogénique du massif cantabrique qui s'est développé, pour l'essentiel, à l'Oligo-miocène.

La plaine alluviale, bien individualisée en contrebas des replats, se compose de trois éléments topo-

(5) G. Bertrano [6]. Cf. bibliographie.

(6) Rañas (espagnol) : plateaux caillouteux; en géomorphologie : nappes caillouteuses jalonnant les plateaux de piémont. 


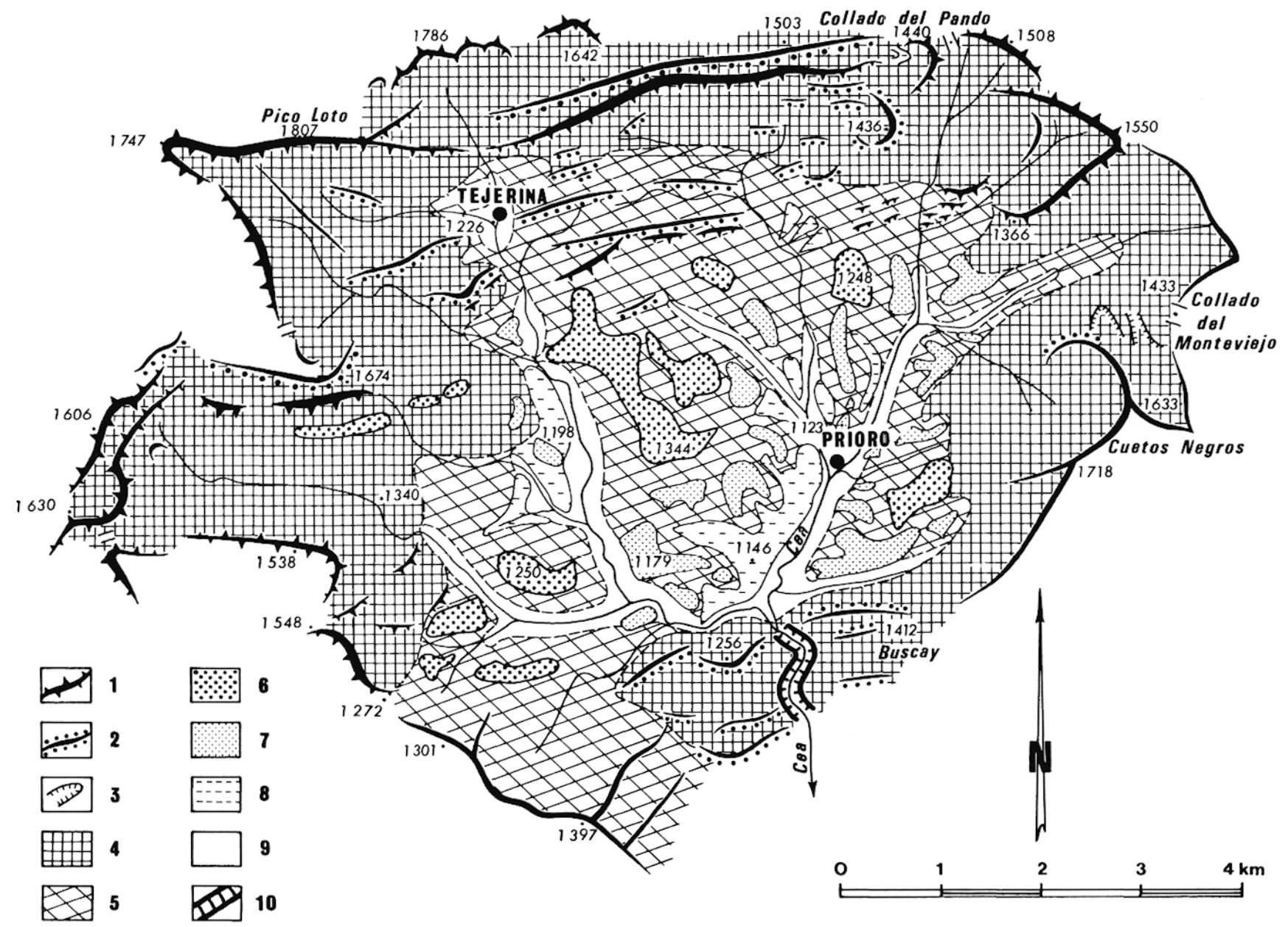

Fig. 2. - Le relief du valle de Prioro.

Crêtes et parois subverticales: 1 . Crêtes et hog-backs dans les calcaires et les conglomérats calcaires du Westphalien. 2. Dans les conglomérats quartzitiques du Stéphanien. - 3. Moraines glaciaires du Maximum. - 4. Versants raides (pente moyenne entre 30 et $50 \%$ ) et très disséqués dans les schistes et les grès tendres. - Versants assez raides (pente entre 15 et $30 \%$ ) et ravinés dans les schistes tendres (faciès $\mathrm{Culm}$ ). - Pentes inférieures à $15 \%$ (dans le même matériel que 5) : 6. Haut niveau des rañas entre 1300-1350 m d'altitude. - 7. Bas niveau des rañas entre $1200-1250 \mathrm{~m}$ d'altitude. 8. Glacis d'érosion du Quaternaire ancien - 9. Vallées alluviales. - 10. Gorges fluviales.

graphiquement et chronologiquement distincts : des glacis-cônes alluviaux très aplatis vraisemblablement préglaciaires ( $W$ du village de Prioro), deux terrasses alluviales dont la plus basse (Maximum glaciaire) forme une nappe épaisse et continue dans laquelle s'encaissent les cours d'eau.

Des talus en roche tendre relaient tous ces gradins topographiques. Entre les crêtes structurales dominantes et les plus hauts niveaux des rañas, de 1600 à $1350 \mathrm{~m}$, s'étirent de longues croupes sculptées par d'étroites vallées. Les roches tendres (schistes et grès), coiffées et raidies par les barres et les crêts durs y sont soumises à de violents affouillements. Les talus plus modestes (20 à $100 \mathrm{~m}$ de commandement) qui séparent les différents niveaux d'aplanissement sont disséqués en roubines (7) et, dans les secteurs de cañadas, en «dos d'éléphant».

(7) Roubine : petit ravin nettement incisé (régions méditerranéennes).
2. Un climat montagneux océanique "continentalisé", à hiver rude et à été sec de type méditerranéen.

Il faut partir de l'étude du bioclimat local qui baigne l'ensemble du Valle de Prioro et pour lequel on possède quelques données météorologiques. Les « topoclimats 》 (J. Tricart), c'est-à-dire les contrastes climatiques liés au système de pente et aux phénomènes corrélatifs d'exposition, et les aspects microclimatiques seront envisagés ultérieurement à l'échelle des géosystèmes et des géofaciès.

Situé sur les marges de l' "Ibérie sèche 》 (H. Lautensach), le Valle de Prioro se rattache pour l'essentiel aux rythmes et aux mécanismes climatiques régionaux de l' «Ibérie humide».

On y reconnaît l'effet orographique direct du versant sud cantabrique au-dessus des plateaux de 
Vieille Castille amorti, il est vrai, par l'éloignement de la mer (circulation cyclonique de secteur SW, à l'automne en particulier); l'effet orographique atténué mais encore efficace de «façade » hyperocéanique propre au versant nord-cantabrique (débordements fréquents des masses d'air océaniques au-dessus des cols de la divisoria par flux de $\mathrm{N}$ et de NE, gros abats d'eau et de neige); l'effet de «continentalisation 》 propre aux bassins du versant sud-cantabrique (assèchement par subsidence de l'air, fort rayonnement et inversions thermiques hivernales); enfin, le développement d'une sécheresse estivale de type méditerranéen (stabilisation anticyclonique), interrompue par de brefs orages orographiques.

Les précipitations sont abondantes mais réparties entre deux saisons pluviométriques tranchées.

L'impression de sécheresse qui se dégage irrésistiblement du paysage doit être objectivement corrigée par l'analyse des séries statistiques de la station météorologique de Prioro. Le centre d'observation de Prioro se situe à $1123 \mathrm{~m}$ d'altitude, au milieu du bassin, en soulane, à la base des grands versants du rebord septentrional (8). La tranche d'eau annuelle
Les séries statistiques imparfaites de la station de Prioro permettent, au plus, de définir un \&climat météorologique moyen $»$. Les bioclimats vrais, plus rudes, plus contrastés, très diversifiés, ne peuvent être appréhendés qu'en combinant l'examen de ces données chiffrées avec les observations quantitatives ou qualitatives réalisées directement sur le terrain (température et humidité de l'air, phénologie, etc.).

La saison végétative est à la fois brève et tardive.

Elle ne dure pas plus de 4 à 5 mois (fin mai-septembre), et elle est de plus perturbée en son milieu par une sécheresse estivale de près de deux mois ( $P<2 \mathrm{~T}$ en juillet-août). Le mois de mai constitue une courte 《charnière » printanière. C'est un mois bien arrosé $(100 \mathrm{~mm})$, aux gelées légères $\left(-2^{\circ}\right.$ à - 4") mais fréquentes, aux chutes de neige rares mais non exceptionnelles, au réchauffement de l'air net quoique irrégulier (moyenne des maximums entre $15^{\prime \prime}$ et $20^{\circ}$ pour une moyenne mensuelle de $12^{\circ}$ ). Le fait écologique majeur est toutefois la fusion nivale qui libère de grosses masses d'eau. Juin est le mois essentiel sur le plan phénologique. Le flux thermique

\begin{tabular}{|c|c|c|c|c|c|c|c|c|c|c|c|c|c|}
\hline & $\mathrm{J}$ & $\mathrm{F}$ & $\mathrm{M}$ & $\mathrm{A}$ & $\mathrm{M}$ & $\mathrm{J}$ & $\mathrm{J}$ & $\mathrm{A}$ & $\mathrm{S}$ & $\mathrm{O}$ & $\mathrm{N}$ & $\mathrm{D}$ & Année \\
\hline $\mathrm{P}(\mathrm{mm})$ & 182.9 & 131.2 & 167.3 & 99.8 & 99.8 & 69 & 30.9 & 32.9 & 67.6 & 116.1 & 180.5 & 184.7 & 1362.7 \\
\hline $\mathrm{T}\left({ }^{\circ} \mathrm{C}\right)$ & $2^{\circ} 5$ & $3^{\circ} 2$ & $6^{\circ} 4$ & $8^{\circ} 2$ & $12^{\circ}$ & $15^{\circ} 8$ & $17^{\circ} 5$ & $17^{\circ}$ & $13^{\circ} 8$ & $10^{\circ} 6$ & $6^{\circ}$ & $2^{\circ} 7$ & $9^{\circ} 6$ \\
\hline
\end{tabular}

moyenne (m) est de $1362 \mathrm{~mm}\left(\mathrm{M}: 1300 ; \mathrm{Q}_{1}: 1000\right.$; $\mathrm{Q}_{2}$ : 1700 ; min. : 712; max.: 2 290) (9). Les contrastes saisonniers sont très accusés. Un puissant maximum de saison froide (maximum principal de novembre à janvier, maximum secondaire en mars) concentre plus des trois quarts des précipitations dont plus de la moitié sous forme de neige. Il s'oppose presque sans transition à un minimum estival court mais creusé (juillet-août).

Les températures, assez basses du fait de l'altitude, présentent de fortes amplitudes saisonnières et journalières.

L'amplitude thermique est forte en toutes saisons (10). Le flux thermique reçu par le Valle de Prioro se caractérise par deux aspects saisonniers très contrastés: d'une part, un refroidissement hivernal précoce (octobre-novembre), brutal, long et profond; d'autre part, un réchauffement estival tardif (juin), puissant (maximum en juillet), mais bref. (8) Station élémentaire d'enregistrement pluvio-thermique
(précipitations : série continue de 34 ans (1931-1965); tempéra(précipitations : série continue de 34 ans (1931-1965); tempéra-
tures : série continue de 4 ans (1960-1963). Les résultats ont tures : série continue de 4 ans (1960-1963). Les résultats ont on a tenu compte de la mise au point critique de J. MounirR (1969) qui a contrôlé et retenu les résultats des trois stations voisines de Riaño, Cistierna et Santibañez de la Peña.

(9) $\mathrm{m}$ : moyenne; $\mathrm{M}$ : médiane; Q1 : $1^{\mathrm{er}}$ quartile; Q2 : $2^{\mathrm{e}}$ quartile, etc.; $\min :$ minimum; $\max$ : maximum. est suffisant (maximums moyens entre 20 et $30^{\prime \prime}$ ). Par contre, les précipitations se répartissent assez irrégulièrement $\left(m: 69 ; M: 65 ; Q_{1}: 35 ; Q_{2}: 93 ; Q_{33}: 23\right.$; $Q_{4}: 130$; min.: 4 ; max.: 189); toutefois, une large compensation s'effectue au niveau des nappes phréatiques du fait de la sursaturation liée à la fusion de la neige. C'est le moment du débourrage général et des floraisons prévernales (en particulier des Géophytes).

Un court été de type méditerranéen, chaud et sec, vient désorganiser l'économie de l'eau et perturber le développement des plantes.

Juillet et août ont toujours $\mathrm{P}<2 \mathrm{~T}$ dans un air sec et fortement insolé :

\begin{tabular}{|l|c|c|c|c|c|c|c|}
\hline \multirow{2}{*}{$\mathrm{P}(\mathrm{mm})$} & $\mathrm{m}$ & $\mathrm{M}$ & $\mathrm{Q}_{1}$ & $\mathrm{Q}_{2}$ & $\mathrm{Q}_{3}$ & \multicolumn{3}{|c|}{ Extrêmes : } \\
\cline { 6 - 8 } & & & & & & $\max$ & $\mathrm{P}=0 \mathrm{~mm}\left({ }^{1}\right)$ \\
\hline Juillet & 30 & 31 & 11 & 45 & 8 & 77 & 3 \\
\hline Août & 32 & 27 & 13 & 49 & 9 & 92 & 1 \\
\hline
\end{tabular}

(1) En nombre d'années sur 34 années d'observation.

(10) Amplitude annuelle extrême : $48^{\circ}$, amplitude extrême pour un même mois d'été (août 1961) : $+32^{\circ}$ et $-2^{\circ} 5=34^{\prime \prime} 5$, id. pour un mois d'hiver (février 1960) : $+30^{\circ}$ et $-16^{\circ}=46^{\circ}$. 
La sécheresse estivale est un phénomène assez stable. Les ressources hydriques ne sont pas suffisantes pour contrebalancer les effets de l'évapotranspiration, surtout si le mois de juin a été sec. Cependant, rien ne permet d'affirmer qu'il y ait interruption du cycle végétatif. Septembre se classe généralement parmi les mois «déficients» $(\mathrm{P}<3 \mathrm{~T})$ mais il est "sec»du point de vue écologique bien que le degré hygrométrique et la nébulosité augmentent, car il succède à une période sèche qui a épuisé les réserves hydriques du sol.

La saison biologiquement défavorable s'étend sur environ sept mois, d'octobre à avril.

L'hiver s'annonce rapidement en octobre et novembre par un très fort accroissement de la nébulosité et une augmentation des précipitations $(116 \mathrm{~mm}$ en octobre, $180 \mathrm{~mm}$ en novembre), dont une partie sous forme de neige ( 4 à 6 jours de chutes de neige en novembre), et aussi par une chute rapide des températures (le cycle gel-dégel devient presque quotidien). Le plein hiver s'établit de la fin novembre à la fin février. Les précipitations bien réparties et surabondantes (décembre $=\mathrm{m}: 184 ; \mathrm{M}: 145 ; \mathrm{Q}_{1}: 83 ; \mathrm{Q}_{2.2}$ : $245 ; Q_{3}: 85 ; Q_{+}: 305 ;$ min. : 32; max.: 572) s'effectuent pour une large part sous forme solide. La couverture de neige se maintient en moyenne plus de trois mois. Les gelées nocturnes sont de l'ordre de -10 à $-16^{\circ}$. En mars et même en avril, la fin de l'hiver se caractérise par un renforcement des précipitations et de la nébulosité qui retarde le réchauffement de l'air et la fusion nivale, c'est-à-dire le démarrage de l'activité biologique. Les facteurs-limitants sont donc multiples et rigoureux. Le bioclimat local $d u$ Valle de Prioro correspond à une fourchette écologique relativement étroite.

\section{Une puissante emprise humaine agro-sylvo-} pastorale a rompu l'équilibre des milieux géographiques et a déclenché une régression généralisée des potentialités écologiques.

Les communautés montagnardes du Valle de Prioro, encore très vivantes et bien structurées, conservent l'entière maîtrise de l'espace rural.

La population s'est régulièrement accrue jusqu'en 1950. Les premières manifestations de l'exode rural n'apparaissent qu'au recensement de 1960 (1900: 985 habitants; $1910: 994 ; 1920: 1079 ; 1930: 1109 ; 1940$ : $1151 ; 1950$ : $1194 ; 1960$ : 1 137). L'équilibre démographique est celui d'une population jeune : en 1950 et 1960 , on a respectivement enregistré 46 et 34 naissances, 21 et 13 décès. La densité moyenne est de 23 habitants $/ \mathrm{km}^{2}$. On compte 240 exploitations agropastorales réparties entre les deux villages de Prioro et de Tejerina. La situation écologique actuelle du Valle de Prioro est comparable à celle des hautes vallées des Pyrénées françaises au milieu du XIx siè- cle. L'exploitation agricole ne laisse pratiquement aucune place à la friche. Les landes et les forêts sont soumises à un pâturage intensif. Il n'y a aucune trace de front pionnier forestier.

Le système pastoral, clé de voûte de la vie économique et sociale, est aussi l'agent écologique qui domine la dynamique actuelle des milieux géographiques.

Le Valle de Prioro abrite l'une des grandes communautés pastorales traditionnelles des montagnes cantabriques. Tout l'espace non cultivé, forestier ou non forestier, est livré au pacage des ovins, des chèvres et des bovins. De plus, on l'incendie régulièrement pour favoriser, pense-t-on, la pousse de l'herbe. Ces vastes pâturages, propriétés communales ou aristocratiques, couvrent plus de $30 \mathrm{~km}^{2}$. Ils sont reliés les uns aux autres et aux villages par de larges cañadas anastomosées. Il y a, d'une part, la vie pastorale et l'élevage locaux qui comportent d'assez gros effectifs (en 1960: 2300 ovins; 912 bovins; 341 chèvres; 59 chevaux, mulets, ânes; soit une densité théorique de 72 têtes de bétail au $\mathrm{km}^{2}$, dont 53 moutons ou chèvres). En excluant la S.A.U. (11) et les parois rocheuses, on dépasse largement une charge animale de 100 têtes au $\mathbf{k m}^{2}$. Seuls les ovins prennent part à la transhumance d'été hors du Valle de Prioro. D'autre part, le Valle de Prioro participe à la grande transhumance castillane, directement héritée de la toute puissante Mesta. Des liens familiaux et économiques rattachent certains éleveurs du Prioro à de grands propriétaires des dehesas (12) d'Extramadura. Bien que le Valle se situe un peu à l'écart des grandes voies de transhumance, il est traversé par une cañada secondaire qui conduit aux puertos pirenaicos (estives) des hauts massifs. Pendant des siècles, le Valle de Prioro a été parcouru et pâturé chaque année, à la montée et à la descente des pâturages d'été, par des milliers de «merinas» de la Mesta. Aujourd'hui, les ovins transhumants ne sont plus que quelques milliers, mais ils suffisent pour maintenir les milieux géographiques en état de surpécoration.

Les forêts, du moins les vestiges délabrés de la couverture forestière, font partie de l'espace pastoral.

Les formations arborées ouvertes, les taillis broussailleux l'emportent sur les futaies qui ne subsistent plus que par lambeaux. Ce sont pour l'essentiel des biens communaux gérés par le Service forestier de l'Etat qui manque de crédits et qui ne porte que peu d'intérêt à ces forêts médiocrement productives. Le projet d'aménagement forestier de 1902 n'a été suivi que de quelques reboisements en Pin sylvestre sur les massifs méridionaux (13).

(11) Surface agricole utile.

(12) Dehesa (espagnol) : pacages extensifs et grands domaines pastoraux.

(13) Proyecto de ordenación de los Montes Buscay et Agragados de Prioro. Servicio forestal del Estado, Provincia de León 1902. 


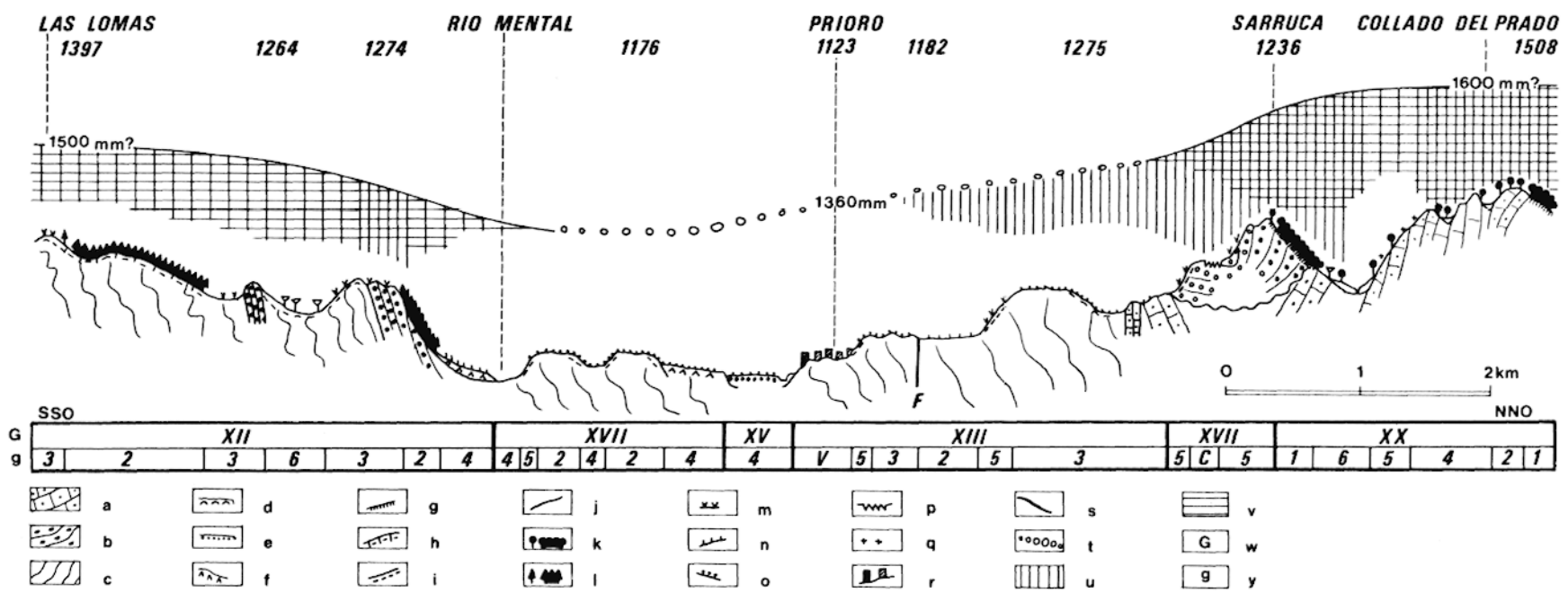

Fig. 3. - Profil synthétique.

a. Calcaires et conglomérats calcaires du Westphalien. - b. Conglomérats quartzitiques du Stéphanien. - c. Schistes tendres (faciès Culm, ardoises, grès schisteux). - d. Glacis préglaciaire. - e. Terrasse fluviatile du Maximum glaciaire. f. Grèze périglaciaire. - $\mathrm{g}$. Sols bruns forestiers et rendzines forestières. - $\mathrm{h}$. Sols bruns acides à pavage superficiel. i. Pavage schisteux de versant. - j. Rankers et lithosols. - k. Hêtre et Hêtraie montagnarde mésophile. - I. Pin sylvestre et Pinède de reboisement. - $\mathrm{m}$. Lande acidiphile ouverte. - n. Pelouse xérophile. - o. Cultures. - p. Cañada (avec roubines). - q. Garide. - r. Village de Prioro. - s. Courbe des précipitations annuelles moyennes. - $t$. Idem: secteur à sécheresse estivale marquée. - u. Nébulosité et degré hygrométrique. - v. Enneigement. - G. Géosystème et géofaciès (les chiffres romains et arabes renvoient au texte).

L'ager s'oppose brutalement au saltus.

L'espace cultivé, compact et bien aménagé, est concentré sur les glacis et les terrasses alluviales du centre-sud du bassin. Encore dominé par le système d'assolement año y vez (14), il paraît très stable dans ses limites. On y cultive du blé, des céréales pauvres et des légumes secs. Les prairies de fauche, entretenues et irriguées, sont cantonnées dans les vallées alluviales.

\section{Un tapis végétal clairsemé et délabré.}

La végétation impose sa spécificité aux paysage; du Valle de Prioro. Une relative richesse floristique contraste avec l'état de démantèlement de l'ensemble du couvert végétal.

Les formations végétales de médiocre venue, dégradées et ouvertes s'étendent sur la presque totalité de l'espace non cultivé.

Les forêts compactes, les pelouses et les landes fermées ne colonisent qu'un petit nombre de stations favorables généralement situées sur les ombrées ou en bordure des thalweg. Les landes couvrent près des deux tiers de la surface totale. Ce sont des formations acidiphiles pauvres, le plus souvent monospécifiques (un Sarothamne ou une Bruyère) et lar-

(14) Año y vez (espagnol) : rotation biennale à jachère. gement vacuolaires, qui n'assurent qu'une médiocre protection au niveau du sol (recouvrement de 5 à $30 \%$ ). Les arbres (Chênes, Hêtres, Alouchiers) qui piquètent les landes ou qui s'associent dans des formations arborées très ouvertes $(10$ à 100 pieds à l'hectare) sont des rejets de vieilles souches. De petite taille $(7-15 \mathrm{~m})$, ramassés et branchus, ils portent dans leur silhouette les traces du vieillissement (cîmes atrophiées ou mortes) et de l'action du vent (port buissonnant ou «en drapeau 》). Le tapis végétal présente deux aspects saisonniers: pendant la mauvaise saison, il se réduit à un peuplement de très faible recouvrement (10 à $50 \%)$ de Phanérophytes isolés (Chênes, etc.) et de Chaméphytes en toufles (Genêts, Bruyères); à la fin du printemps, la couverture se densifie avec la pousse des Géophytes et des Thérophytes mais le sol n'est jamais entièrement couvert ( 20 à $70 \%)$. La seule exception, significative sur le plan écologique et pastoral est, au printemps, l'expansion des grandes Fougeraies à Fougère-Aigle qui verdissent les terrains de parcours préalablement incendiés.

Les formations végétales s'étagent irrégulièrement le long des versants de 1100 à $1800 \mathrm{~m}$ d'altitude.

On a distingué quatre séries végétales qui se rattachent à trois étages de végétation.

L'étage continental-montagnard n'est représenté que par la série acidiphile du Chêne Tauzin qui occupe le centre de la dépression $(1100-1300 \mathrm{~m})$ sous la forme de taillis dégradés de 5 à $20 \mathrm{~m}$ de haut, clairs et envahis par les espèces héliophiles de la lande: Chêne Tauzin (Quercus pyrenaica Wild ou $Q$. 
toza Bosc) (15), Aubépine (Crataegus oxyacantha L.), Sarothamne (Sarothamnus cantabricus Wk.), Bruyères (Erica arborea L., E. mediterranea L., E. umbellata L.). Les pelouses et les friches se caractérisent surtout par: Santoline (Santolina pectinata Lag.), Thyms (Thymus mastichina, T. Chamaedrys Fries), Centaurées (Centaurea paniculata L., C. solsticialis L.), Plantains (Plantago carinata Schard ..., etc.).

L'étage montagnard inférieur, entre $1250 \mathrm{~m}$ et $1450 \mathrm{~m}$, est colonisé par la série acidiphile du Chêne Sessile fortement hybridé avec le Chêne Tauzin ou le Chêne Pédonculé (Quercus petraea (Matts) Lieb). La strate arborée, clairsemée et monotone, ne comporte pratiquement que de vieux chênes; la lande couvre pratiquement tout. On doit ajouter aux espèces précédemment citées: Genêt cendré (Genista cinerea DC), Bruyères (Erica aragonensis WK ou $E$. australis L., E. lusitanica ssp. Rudolphi, E. vagans L.), Airelle (Vaccinium myrtillus L.), Fougère-Aigle (Pteris aquilinum (L) Kuhn), Jasione (Jasione montana L.), etc.

L'étage montagnard supérieur, entre 1400 et 1650 $m$ d'altitude, se limite pratiquement aux massifs calcaires. Il est représenté par la série calcicole et mésophile du Hêtre (Fagus silvatica L.). Les quelques massifs forestiers sont des Hêtraies soit pures, soit ponctuées de Chênes divers. On y trouve la plupart des espèces caractéristiques des sous-bois ombreux et humides de la Hêtraie pyrénéo-cantabrique : Asperule odorante (Asperula odorata L.), Anémone Sylvie (Anemone nemorosa L.), etc. L'Alouchier (Aria nivea (L) Crantz), se mêle au Hêtre sur les lisières forestières et dans les formations arborées ouvertes. Le faciès régressif le plus caractéristique est représenté par une garide calcicole xérophile: Thyms (Thymus Chamaedrys Fries et T. mastichina, etc.), Germandrées (Teucrium Polium L., T. Chamaedrys L.), Hélianthème (Helianthemum alyssö̈des Vent.), Ononis (Ononis spinosa var.), Epipacte (Epipactis latifolia All.), Kœleria (Kœleria Valesiaca Gaud).

L'étage subalpin, difficile à délimiter, est constitué de landes acidiphiles qui ne se différencient pas toujours des landes paraclimaciques de l'étage montagnard. Le Sarothamne cantabrique, la Myrtille, la Bruyère-Callune forment le fond du peuplement. Il s'y mêle: Genêts (Genista obtusiramea Gay et G. tridentata L. ou trichomanes), Thym (Thymus alpestris Briquet), Millepertuis (Hypericum Burseri Spech.), Astragale (Astragalus sempervirens Lamk), Erodium des pierres (Erodium petraeum Willd)...

La mosäqque végétale du Valle de Prioro, par sa diversité floristique et son degré de démantèlement, pose deux problèmes essentiels du point de vue de la dynamique globale des paysages.

(15) Le Tauzin qui a une aire eu-atlantique en France est, dans le Nord-Ouest de la Péninsule Ibérique, une plante des montagnes sèches à nuance continentale (espèce atlantique ibéro-montagnarde de $P$. DuPoNr, $L a$ flore atlantique européenne. Document pour les cartes des productions végéeuropéenne. Document pour les cartes des prod
tales. Toulouse, Thèse Sciences, 1962, p. 285).
Tout d'abord, la forêt n'a jamais dû recouvrir l'ensemble des étages continental et montagnards. On ne doit pas raisonner comme si le climax était, partout, de type forestier. Même si on exclut l'étage subalpin s.s., la forêt n'a jamais colonisé certains versants audessus de 1450-1550 m d'altitude. Les facteurs limitants sont, d'une part, le développement des parois subverticales et les larges affleurements de conglomérats quartzitiques hyperacides; d'autre part, en l'absence de conifères montagnards et subalpins, ce sont le Hêtre et l'Alouchier, espèces de l'étage montagnard «moyen», qui forment la limite supérieure de la forêt [1].

Ensuite, le voisinage brutal entre des formations végétales peu dégradées à base de plantes «humides » (Hêtraies à Anémone Sylvie, Chênaies à Chêne Pédonculé) et certains faciès plus dégradés, où le caractère xérophile s'accompagne d'un renouvellement presque complet du stock floristique, ne peut pas s'expliquer par une simple "régression》 des séries végétales. Ne s'agirait-il pas là d'un trait acquis récemment sous l'effet de la mise en valeur anthropique qui, par "assèchement» des milieux, aurait entraîné une colonisation par des plantes «xérophiles»?

\section{Un complexe édaphique et hydrologique dé- gradé et instable.}

L'épiderme des versants du Valle de Prioro porte sur plus de la moitié de sa surface les traces d'une géomorphogénèse active dominée par les différentes formes du ruissellement et de la fragmentation des roches. De grands plans inclinés en roche nue parsemés de guirlandes de dépôts meubles, des ravines vives qui lacèrent les talus des glacis, des vallons secondaires bourrés de formations pédogénisées voisinent avec des versants forestés qui présentent toutes les apparences de la stabilité. L'érosion n'est pas ici un phénomène généralisé de type climacique. Elle est, au contraire, la marque du déséquilibre écologique actuel du Valle de Prioro.

Un écoulement à caractère torrentiel dans un petit bassin-versant $\left(49 \mathrm{~km}^{2}\right)$ en roches imperméables et tendres, sans réserves hydrologiques.

Le rio Cea draine un bassin taillé dans des schistes feuilletés rapidement débités en plaquettes et des grès-quartzites fortement diaclasés. Le calcaire à faciès conglomératique n'affleure que sur 15 à $20 \%$ du bassin-versant et les formes karstiques sont très localisées et mal développées (des lapiés et quelques avens). Il n'y a pas de réserves hydrologiques profondes. Le ruissellement, qui se concentre à partir de couloirs torrentiels à profil longitudinal tendu, répercute instantanément les forts abats d'eau et les eaux de fusion nivale. En l'absence de mesures et d'observations saisonnières cohérentes, on peut admettre le déroulement du régime suivant: étiage estival très 
Pour le commentaire, se reporter au texte. Les figures et les symboles sont les mêmes que ceux du texte et des cartes.

Ci-contre. - Le nord-est du Valle de Prioro vu du centre du bassin : la mosaïque des trois grands géosystèmes du Valle de Prioro et de huit de leurs géofaciès.

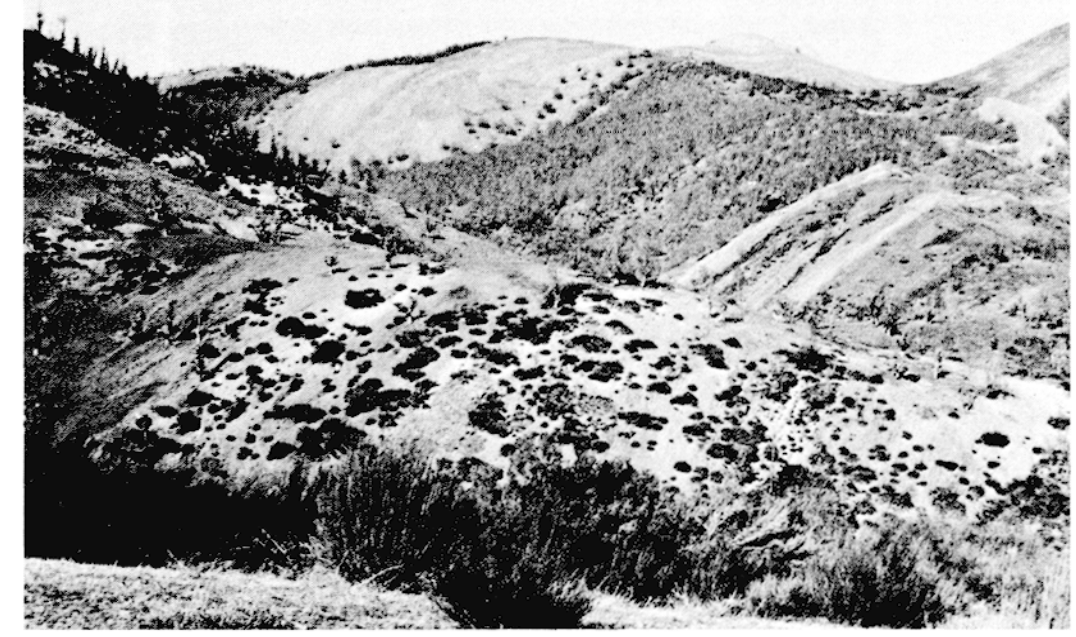

Ci-dessous. - Le nord-ouest du Valle de Prioro vu du centre du bassin. Au premier plan, une «cañada» secondaire qui se détache de la « cañada» principale au niveau du village de Prioro.
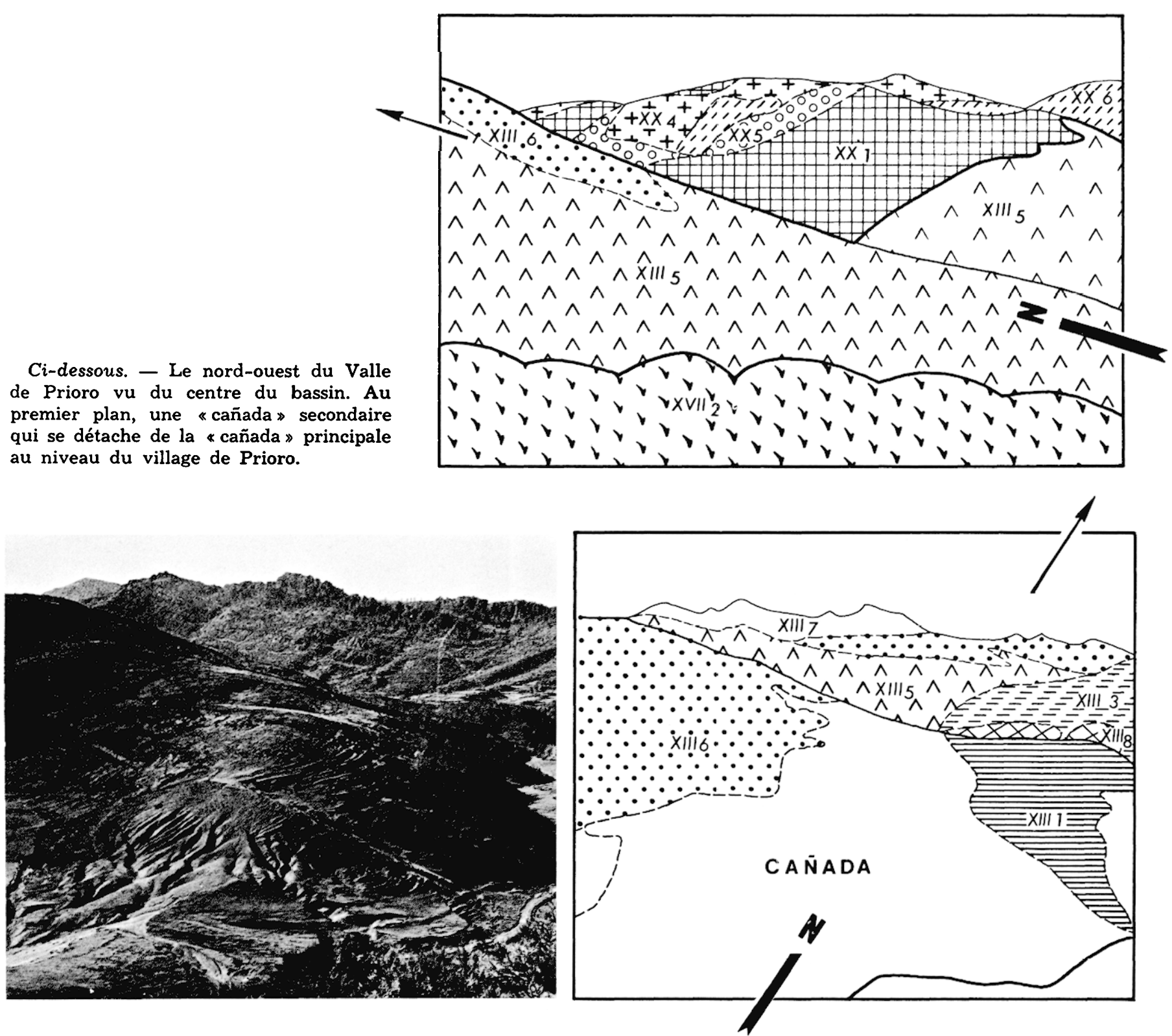
prononcé avec les vallons secondaires à sec (juilletaoût), maximum secondaire d'automne avec crues pluviales instantanées souvent à la suite d'orages (septembre-novembre), rétention nivale de période froide (décembre-mars), maximum principal lié à la crue de fusion nivale et aux copieuses pluies de printemps (avril-mai). Le déséquilibre saisonnier, et plus particulièrement la pénurie estivale, sont encore accusés par la très faible rétention superficielle des manteaux de débris et des sols.

Des versants hérités du Quaternaire «froid», démantelés et disséqués par le ruissellement actuel.

A l'exception des trois niveaux de glacis, des terrasses alluviales et des dépôts morainiques de hauteur, les pentes ont été modelées par les processus périglaciaires: versants réglés, nappes gréziformes, coulées de solifluction colmatant les vallons. Tous ces modelés sont abâtardis uu démantelés au sens propre du terme par des processus postérieurs qu'il faut rattacher à un système morphogénique anthropique. Les dépôts meubles sont mobilisés et la roche en place est fragmentée en débris grossiers, sans fournir beaucoup d'éléments sableux ou colloïdaux (plaquettes de schistes, galets quartzitiques dégagés du ciment des poudingues). C'est l'origine des nombreux pavages de versant.

Les versants nus, sans "couverture vivante 》, c'est$\grave{a}$-dire sans végétation et sans sol, couvrent plus $d u$ tiers de la superficie du Valle de Prioro.

Une typologie sommaire permet d'isoler les parois taillées dans les conglomérats (calcaires ou quartzitiques) qui sont généralement des éléments de crêts ou de barres, les versants rectilignes de dénudation (schistes ou versants structuraux dans le calcaire), les croupes convexes à pavage mobile, les roubines qui ourlent les replats de glacis. La conjonction «talus-roche tendre - dépôts meubles - exposition sud» est un fait écologique essentiel, responsable de la concentration géographique des phénomènes de météorisation.

\section{Les sols sont minces et discontinus.}

Les sols in situ de plus de $30 \mathrm{~cm}$ d'épaisseur et à profil complet ne se rencontrent que sous les forêts et les landes denses. Sur calcaire et sous la Hêtraie, se développent des rendzines ou des sols bruns calcaires; les landes acidiphiles s'équilibrent avec des sols bruns lessivés. Sous ce climat relativement humide, il semble que la brunification et le lessivage soient les processus pédogénétiques dominants. Les sols AC, de type Ranker, couvrent un tiers environ de la surface du Valle de Prioro. Ils sont parfois fossilisés par un pavage de schistes. La géomorphogénèse l'emporte ici sur la pédogénèse. Les sols "hérités » en place sont rares, par exemple une tourbière à Sphaignes, relictuelle, partiellement décapée et perchée au-dessus des pavages actuels. Le qualificatif de sol «hérité» s'applique aussi à tous les manteaux de débris pédogénisés dans leur masse, vraisemblablement d'anciens sols bruns décapés, qui bourrent les fonds de vallon ou que l'on trouve, en transit, sur les hauts versants sous forme de guirlandes nivales. Ils constituent les seuls réservoirs aquifères superficiels.

Le déficit hydrique de saison sèche est le principal «facteur écologique limitant» des milieux géographiques du Valle de Prioro.

L'eau qui est stockée en hiver sous forme de neige est rapidement évacuée au printemps comme l'est l'eau pluviale après chaque averse. La capacité de rétention est très limitée. Les rares nappes phréatiques épidermiques sont des nappes perchées et isolées (remblaiement de vallon, loupe de solifluction), vestiges d'anciennes nappes plus vastes et mieux alimentées qui ont été morcelées par l'érosion. L'ablation d'une grande partie des dépôts meubles et la désorganisation consécutive des niveaux phréatiques a entraîné une multiplication des petits points d'émergence (suintements, sourcins) qui accélèrent le tarissement. Les sources pérennes sont rares et de débit médiocre. La «sécheresse » des paysages du Valle de Prioro est donc un fait hydrologique d'origine récente beaucoup plus qu'un trait climatique et climacique.

\section{L'ETTUDE INTÉGREE DES MILIEUX GÉOGRAPHIQUES}

\section{La méthode globale.}

L'inventaire des données par grands secteurs (relief, climat,...) ne constitue pas une chaîne explicative logique appuyée sur une description raisonnée des paysages. C'est un cheminement nécessaire mais insuffisant. Les milieux géographiques du Valle de Prioro ne sont traités ni en eux-mêmes, ni pour euxmêmes. "Le cloisonnement fait office d'ordre, mais point d'intelligibilité » (16).

L'étude des paysages du Valle de Prioro est un essai d'application d'une théorie de la méthode géographique globale qui a été exposée par ailleurs et dont on ne reprendra que les conclusions indispensables (17).

L'objet-paysage considéré comme un «ensemble» géographique indissociable est une réalité qui transcende les analyses sectorielles. Il faut recourir à une méthode globale, c'est-à-dire, en un sens, «structu-

(16) Michel Bouet, in Le Monde, Paris, 21 avril 1971, p. 25.

(17) G. Bertrand [3], Voir aussi : Colloque sur la « Science du paysage et ses applications ». Institut de Géographie de Toulouse (mars 1970); à paraître dans un numéro spécial de la Revue Géographique des Pyrénées et du Sud-Ouest (1972). 
raliste ». Comme toute structure, le paysage est formé d'éléments "mais ceux-ci sont subordonnés à des lois caractérisant le système en tant que tel; et ces lois de composition ne se réduisent pas à des associations cumulatives, mais confèrent au tout en tant que tel des propriétés d'ensemble distinctes de celles des éléments 》 (18).

L'espace géographique est discontinu. C'est une mosaïque d'unités spatiales isomorphes dont l'homogénéité est un caractère relatif lié à l'échelle (10 ha de soulane calcaire à garide, 200 ha de collines schisteuses colonisées par des taillis de Chêne Tauzin).

Chaque unité constitue, à son échelle, une structure spatiale, c'est-à-dire une combinaison d'éléments hiérarchisés et interdépendants. Pour plus de commodité, on a distingué trois sous-ensembles: le potentiel abiotique (roche, air, eau), l'exploitation biologique (végétaux et animaux), l'utilisation anthropique (incendie, défrichement, culture, etc.).

Chaque structure spatiale isomorphe se définit par une dynamique propre. Celle-ci est déterminée par les interactions de tous les éléments du complexe au sein d'un «système d'évolution » propre à l'unité géographique considérée. Il englobe le système géomorphogénique, le dynamique biologique et le système de mise en valeur socio-économique.

Chaque unité dynamique est douée d'un certain degré de permanence: une ombrée forestière est plus stable qu'une soulane surpâturée.

Les unités de paysage sont liées les unes aux autres par des rapports dynamiques qui jouent dans le temps et dans l'espace. Il faut donc classer les unités les unes par rapport aux autres en fonction de leur structure, en particulier de leur taille, et de leur dynamique, donc de leur permanence. La taxonomie proposée n'est, en fait, qu'une chorologie. En théorie, elle comporte six niveaux spatiaux principaux : la zone, le domaine géographique, la région naturelle, le géosystème, le géofaciès et le géotope.

La taxonomie écologique du Valle de Prioro doit être considérée à deux niveaux différents. D'une part, l'insertion écologique dans le Nord-Ouest ibérique : le Valle de Prioro se rattache au domaine montagnard cantabrique qui s'étire entre l'Océan atlantique et la Vieille Castille, de l'Asturias aux Provinces basques. Le versant sud cantabrique, au relief aéré et au climat plus sec, constitue un sous-domaine différent $\mathrm{du}$ versant nord plus compact et plus océanique. Le versant sud cantabrique se divise à son tour en trois régions naturelles: les Hautes régions de la divisoria, les Sierras calcaires méridionales et, entre les deux, la Dépression intramontagnarde dont le Valle de Prioro est une sous-région.

D'autre part, les divisions écologiques internes du Valle sont plus complexes. L'opposition déjà signa-

(18) Jean Piaget, Le structuralisme. Paris, P.U.F., 1970. Coll. Que sais-je?, n ${ }^{\circ} 1311$, p. 8. lée entre un Haut et un Bas Prioro n'est qu'une première impression liée à une "lecture » directe et rapide du paysage. Chaque analyse sectorielle fait apparaître un découpage spécifique de l'espace (fig. 2). La superposition cartographique de l'ensemble des divisions sectorielles ne peut donner que des contours grossiers et souvent inexacts. La cartographie intégrée a été pratiquée directement, soit sur le terrain, soit par photo-interprétation (fig. 3).

Le Valle de Prioro se subdivise en cinq géosystèmes (19) : le géosystème supraforestier «acide» (G. XXVIII); le géosystème montagnard «calcaire 》 (G. $\mathrm{XX}$ ); le géosystème semi-montagnard «acide» (G. $\mathrm{XIII}$; le géosystème continental-montagnard « acide» (G. XVII); le géosystème continental «acide» (G. XV). On laissera provisoirement de côté $G$. XXVIII et G. XV qui sont deux unités peu étendues et marginales par rapport au Valle de Prioro.

\section{Le géosystème montagnard calcaire du Haut Prioro (G. XX).}

C'est l'ensemble géographique le mieux individualisé du Valle de Prioro. D'ailleurs, il se rattache autant à la «région naturelle » des Sierras calcaires méridionales qu'à la Dépression intramontagnarde. Etendu sur une quinzaine de kilomètres carrés, soit près du tiers de la surface du bassin, il englobe à la fois les massifs du nord-est (col del Pando) et les contreforts montagneux du sud-ouest (entre 1400 et $1670 \mathrm{~m}$ d'altitude). La combinaison écologique est dominée par quatre éléments qui interfèrent de façon originale : le climat montagnard le plus arrosé (1 400-1500 mm au minimum), le plus enneigé (environ 3 mois de couverture neigeuse) et le plus nébuleux du bassin; l'unique substrat calcaire disséqué en parois et versants raides commandés par la structure (barres et crêts, pentes substructurales de revers); l'unique Hêtraie qui présente, il est vrai, un certain nombre de caractères floristiques et écologiques marginaux; situé au-dessus de la zone cultivée, ce géosystème est au cœur du secteur sylvo-pastoral et ses paysages inégalement dégradés portent la marque de cette double mise en valeur qui a accentué les contrastes entre les versants.

Le géosystème $\mathrm{XX}$ est caractérisé par des unités isomorphes d'assez grande taille, entre 500 et 3000 ha. La distribution des géofaciès, on en a distingué sept, est essentiellement commandée par les effets combinés des topoclimats, de la charge pastorale et de la pente.

$X X-g 1$ : Les ombrées à Hêtraie sur sol brun représentent la structure écologique la plus com-

(19) G. Bertrand, Les géosystèmes cantabriques (avec une carte en couleurs au 1/200 000). Revue géogr. Pyrénées $S .-O$. (1972). La classification des géosystèmes repose sur une taxonomie systématique des unités géographiques des montagnes cantabriques (G.I, G. II... : géosystèmes; g1, g2 : géofaciès; $\gamma 1,2 \ldots$ : géotopes). 


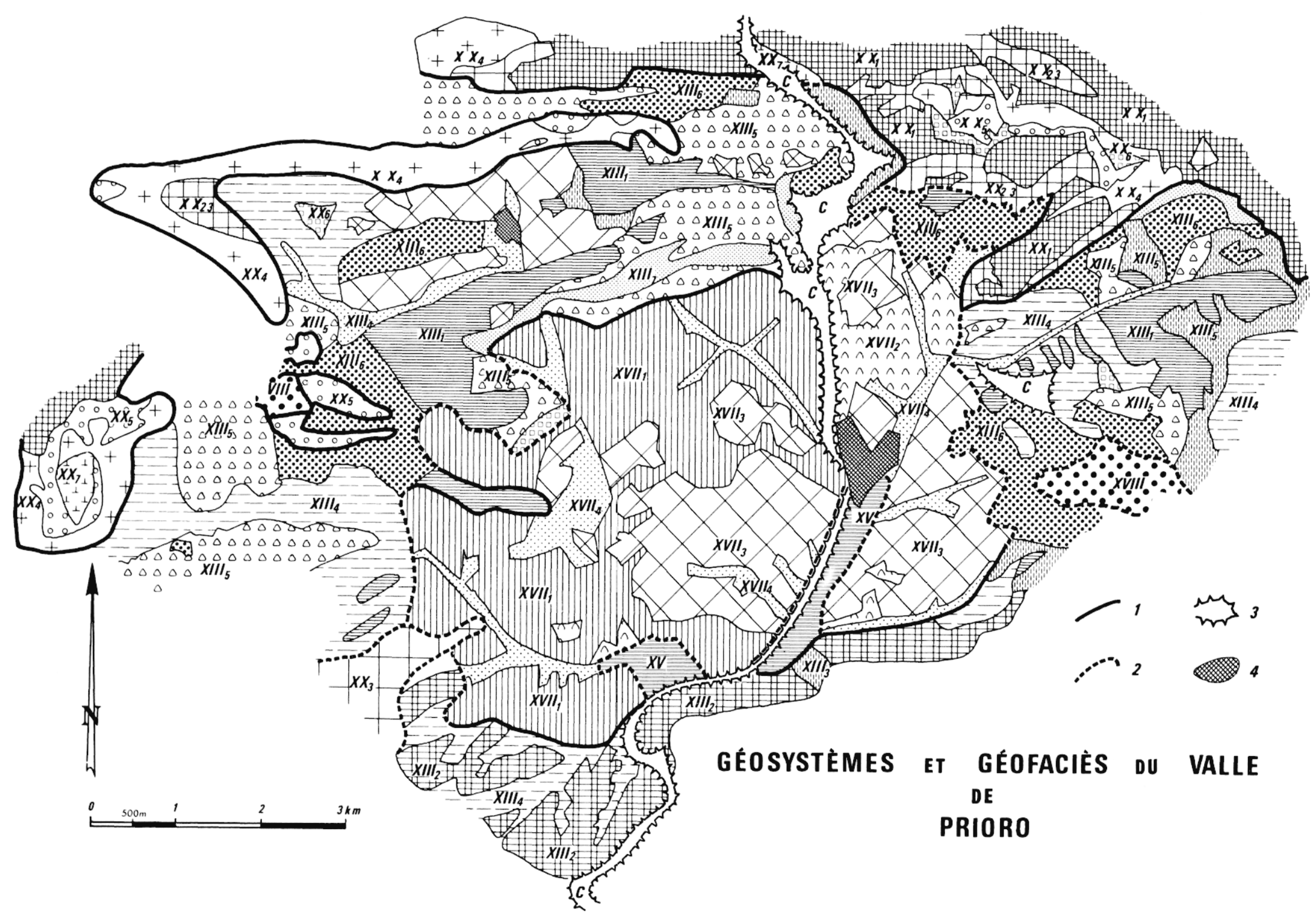

Frg. 4. - Esquisse cartographique des géosystèmes et des géofaciès du Valle de Prioro.

Les contours ont été schématisés et certains géofaciès ont été regroupés; le fond utilisé est celui des photographies aériennes au 1/28 000-1/30 v00, non restitué. - 1. Limite de géosystème nette et stable. - 2. Limite de géosystème instable et peu marquée. - 3. Cañada principale (voie de transhumance). - 4. Villages.

La légende détaillée de la carte correspond au deuxième chapitre de l'article (les chiffres romains renvoient aux géosystèmes et les chiffres arabes aux géofaciès). 
plexe, la plus stable et la moins éloignée du climax de tout le Valle de Prioro (20).

Ce géofaciès forestier se développe aussi bien sur substrat calcaire que siliceux. Mais il reste toujours circonscrit aux ombrées. Stable sur le plan géomorphogénique, il constitue la plus importante biomasse du Valle de Prioro et représente vraisemblablement la meilleure productivité en matière végétale (en particulier pour les arbres). Toutefois, la régénération naturelle par graine du Hêtre paraît impossible. Le recépage est vigoureux mais bloqué à l'état arbustif. Le géofaciès des ombrées forestières est en état de biostasie, mais l'équilibre biologique en est précaire. Se maintenant par inertie, il ne se reconstitue pas après destruction.

$X X-g 2$ : Les replats à Hêtraie-parc et XX-g3 : Les versants doux à pelouse arborée. Ces deux géofaciès sont en équilibre avec la vie pastorale actuelle. Les replats à Hêtraie-parc se limitent aux surfaces d'interfluve dans les schistes qui s'ouvrent en cols entre les alignements de crêts et de barres calcaires. Ce sont des «cañadas» obligatoires pour les troupeaux. La Hêtraie y perd sa structure forestière et ses éléments floristiques les plus caractéristiques (Anémone, Aspérule, etc.) Les arbres, en pieds isolés tous les $15-30 \mathrm{~m}$ ou en bouquets de 3-4 tiges originaires d'une même souche, ont le port en boule caractéristique $(10-20 \mathrm{~m}$ de haut, première fourche à 5-10 $\mathrm{m}$ du sol, diamètre des troncs : 0,80 à $1,30 \mathrm{~m}$ pour 70-90 ans d'âge). Il n'y a aucune régénération. $\mathrm{La}$ lande associée comporte deux étages: une strate sous-arbustive dominée par la Fougère-Aigle (3-4) et parsemée de Sarothammes et de Ronces; une strate herbacée à Fétuques (4-5). Les sols formés dans un mince régolithe schisteux sont proches de ceux qui existent sous forêt; cependant, les horizons humifères sont plus minces et l'ensemble du profil est plus tassé et plus argileux. L'absence de pente, le couvert végétal continu en toutes saisons (en hiver les touffes persistantes de graminées et les hampes desséchées des Fougères protègent le sol d'un contact direct avec la neige), ie développement du système racinaire de la Fougeraie, contribuent à assurer la stabilité de ce géofaciès malgré l'importance de la charge pastorale.

(20) Relevé-type : Station à $1450 \mathrm{~m}$ d'altitude, à mi-versant, sur une pente de $35 \%$, exposition $\mathrm{N}-\mathrm{NE}$, dans l'ombre portée des massifs septentrionaux, face au col del Pando qui est fréquemment débordé par les masses d'air humides et nébuleuses: 1) forêt claire (recouvrement 90-95\%): strate forestière : Hêtre 3-4 (les chiffres qui suivent un nom de plante correspondent à un relevé d'abondance-dominance - de 0 à $5-$ ) $20-25 \mathrm{~m}$ de hauteur, fût mal dégagé, croissance lente; taillis évoluant vers une médiocre futaie; sous-bois : Plantules de Hêtre +, Aspérule odorante 1, Anémone Sylvie +; litière continue, épaisse $(5 \mathrm{~cm})$, bien décomposée à la base. Glissements lents de la litière, quelques terrassettes liées au passage du bétail. - 2) sol brun argileux, souple, $50-70 \mathrm{~m}$ d'épaisseur, bonne pénétration des racines; horizon $B$ faiblement marmorisé par place. - 3) nappe phréatique régulièrement alimentée et proche de la surface (ligne de source). - 4) versant schisteux faiblement convexe; coulée de solifuction de 2 à $3 \mathrm{~m}$ d'épaisseur (fines plaquettes de schistes et éclats calcaires) entièrement stabilisée. - Les chiffres qui suivent un nom de plante correspondent à un relevé d'abondance-dominance (de 0 à 5 ).
Les versants doux à pelouse arborée présentent les mêmes caractères de paraclimax. Le pacage intensif et le piétinement du bétail y entretiennent une pelouse rase de graminées xérophiles (Kœleria Valesiaca Gaud...) en excluant Fougères et Genêts. Les sentes sont soulignées par des micro-formes de versant, terrassettes et buttes gazonnées, qui paraissent stabilisées.

XX-g4: Les soulanes décapées à garide et $X X-g 5$ : Les soulanes rocheuses arborées couvrent plus du tiers de la surface du géosystème. Leurs paysages dévégétalisés, décharnés, les opposent violemment aux ombrées forestières qui leur font face (21).

Les soulanes rocheuses arborées se développent uniquement sur le revers structural des crêts calcaires. Sur des pentes entièrement rocheuses de 40 à $60 \%$ se dressent quelques arbres isolés, Hêtres et Alouchiers, pas plus de 10-20 pieds par hectare (enracinement direct dans les diaclases, fûts de 5-10 m pour une hauteur totale de $12-20 \mathrm{~m}$, silhouette intermédiaire entre le port en boule et le port forestier, aucune régénération ni par graine ni par rejet, âge de 80-100 ans. Il n'y a pas d'autre végétation et aucun sol en place. Par contre, la soulane rocheuse est ornée de guirlandes de dépôts pédogénisés qui résultent de l'action actuelle du ruissellement et de la neige. Ce paysage est tout le contraire d'un front pionnier forestier. Il faut admettre que ces arbres se sont développés dans un milieu de forêt claire et sur des sols continus. Il s'agit donc d'un géofaciès de régression récente, une centaine d'années au maximum, lié à la surpécoration. L'érosion actuclle cst faible car tout le matériel meuble a été évacué. Il ne se forme actuellement que quelques petits éboulis de gélifraction. Les unités g4 et g5 marquent donc le terme d'une évolution rhexistasique liée aux effets combinés du topoclimat de soulane et de la vie pastorale.

XX-g6 : Les vallons remblayés à pelouse xérophile. Les manteaux d'altération arrachés aux soulanes sont, pour partie, bloqués au pied des versants et dans les vallons latéraux où ils forment des accumulations discontinues de plusieurs mètres d'épaisseur. Ces dépôts meubles, généralement formés par de la grèze calcaire mélangée à des horizons de sols bruns rendziniformes, sont ravinés et prennent localement l'aspect de roubines. Ailleurs, ils sont plus ou moins protégés par une pelouse xérophile dense mêlée de plantes à fort enracinement : Fougère-Aigle, Thyms, Bruyère méditerranéenne.

$X X-g 7$ : Les soulanes à lande acidiphile ne sont pas uniquement liées aux affleurements et aux pava-

(21) Le relevé-type de la soulane décapée à garide se situe à $150 \mathrm{~m}$ de distance du relevé $\mathrm{XX}-\mathrm{g} 1$ : station à $1480 \mathrm{~m}$ d'altitude, à mi-versant, pente de $25 \%$, exposition S-SW : garide ouverte (recouvrement : $5 \%$ ), monostrate (Cf. ci-dessus I 4, c); quelques lithosols, pas de nappe phréatique; versant réglè périglaciaire à champs de lapiès émoussés, quelques éboulis actifs. 
ges de roches acides. A partir de ceux-ci, la lande colonise par «tache d'huile» les soulanes calcaires découvertes grâce à la plasticité de ses composants (Ericacées et Sarothamne). Cette évolution encore limitée à quelques dizaines d'hectares n'en indique pas moins l'amorce de la régression du géosystème montagnard calcaire au profit du géosystème semimontagnard « acide ».

\section{Le géosystème semi-montagnard "acide" du Moyen Prioro (G. XIII).}

Les milieux qui s'intercalent entre les bastions calcaires périphériques et les paysages horizontaux de la cuvette centrale forment un ensemble de transition bien qu'ils soient physionomiquement très proches des paysages du Bas Prioro. Ce géosystème dessine une ceinture presque continue entre 1200 et $1400 \mathrm{~m}$ d'altitude (mais il peut s'élever jusqu'à $1600 \mathrm{~m}$ ). Il couvre environ $18 \mathrm{~km}^{2}$, soit plus du tiers de la surface totale. La combinaison écologique de base est assez banale :

- un milieu acide. L'équilibre climacique repose pour partie sur les interrelations entre l'acidité des roches (schistes, grès), des sols (bruns acides, rankers), des eaux superficielles et de la couverture végétale;

- c'est le domaine par excellence de la lande acidiphile (Callune, Ericacées, Genêts, Sarothamne). Les formations végétales, très pauvres, sont monotones et souvent monospécifiques. La Chênaie climacique, un mélange de Chênes Sessile, Pédonculé et Tauzin, a été largement détruite;

- un rythme bioclimatique contrasté où les fortes précipitations de saison froide alternent avec une période de sécheresse estivale très marquée. Les topoclimats jouent un rôle essentiel dans la distribution des géofaciès mais d'une façon moins systématique qu'à l'intérieur du géosystème montagnard calcaire (fig. 4);

- des versants en rhexistasie. Les défrichements agricoles et pastoraux ont avivé une érosion qui se développe dans des conditions géomorphologiques favorables : longues pentes raides en roche tendre (faciès Culm), début de la concentration et de l'incision torrentielle.

XIII-g1 : Les ombrées et les ravins forestés ne se rencontrent que dans les vallées de l'ouest et du nord, là où l'effet topoclimatique est accentué par l'advection des masses d'air humides et nébuleuses qui débordent les cols septentrionaux (22).

(22) Relevé-type : $1350 \mathrm{~m}$ d'altitude, base d'un versant, pente $20 \%$. - 1) Forêt claire colonisée par la lande (recouvrement : $60-70 \%)$ avec : Strate forestière $(20 \%)$ : Chêne hybridé (Q. Petraea) (3), Hêtre + , Taillis $15-20 \mathrm{~m}$ de hauteur, âge $80-100$ ans; lande associée $(60 \%)$ : Bruyère méditerranécone (3), Bruyère cendré (1), Genêt Cendré (1) Gé richomanes (1), Houx (1). 2) sol brun : 20-30 cm d'épaisseur, acide ( $\mathrm{pH} 5)$, sec; roche-mère : plaquettes de schistes emballées dans de l'argile; le sol est généralement surmonté
Ce n'est plus un milieu forestier mais un complexe de dégradation post-forestier. La biomasse, moins importante que celle de la Hêtraie d'ombrée, ne paraît pas devoir se maintenir. La régénération, nulle pour l'hybride Sessile-Pédonculé, faible pour le Tauzin, est un peu plus favorable pour le Hêtre qui, bien que subordonné dans la strate forestière, rejette mieux de souche et résiste davantage à l'incendie et au pacage. Cela ne signifie pas que le Hêtre se substitue aux Chênes et, encore moins, la Hêtraie à la Chênaie.

$X I I I-g 2$ : Les plantations en Pin sylvestre. Réalisées dans les Chênaies claires des massifs méridionaux, elles se développent dans d'assez bonnes conditions sans modifier des sols et un sous-bois déjà très acides. On note seulement un épaississement de la litière et un éclaircissement des sous-bois. Des coupefeux protègent ces nouvelles forêts qui sont très menacées par les incendies volontaires de landes et de broussailles voisines.

XIII-g3 : Les croupes à landes basses et XIII-g4 : Les versants à pelouses denses correspondent à des milieux régulièrement pacagés. La lande monospécifique à Callune s'étend au dépens des autres formations. Elle s'équilibre avec des Rankers ou des sols bruns lessivés. Le sol est relativement bien protégé contre l'érosion mécanique.

XIII-g5 : Les versants raides à pavage mobile associés soit aux landes hautes, soit (XIII-g6) aux formations arborées très claires constituent les unités géographiques les plus originales et les plus étendues de ce géosystème. Dans le cas de la lande haute, la strate arbustive dominante a un taux de recouvrement de 25-30\% à 1-2 $\mathrm{m}$ au-dessus du niveau du sol. Elle se présente presque toujours comme un peuplement monospécifique (Sarothamne cantabrique, Bruyère méditerranéenne, Bruyère arborescente, etc.). Il s'agit toujours de plantes acidiphiles, sociables, à enracinement profond et résistantes à la sécheresse. Le sol est nu entre les touffes. Les pavages mobiles, épais de 3 à $20 \mathrm{~cm}$, reposent directement sur le schiste en place, ou fossilisent des dépôts fins pédogénisés qui ne sont peut-être pas des sols «hérités » et tronqués, mais plus vraisemblablement des sols actuels qui continuent à évoluer sous le pavage protecteur. Les quelques Chênes Tauzin multicentenaires, au troncs creux et mutilés, ne modifient pas la dynamique du versant. Ce sont les vestiges d'une ancienne couverture arborée ... mais certainement pas forestière.

$X I I I-g 7$ : Les parois rocheuses taillées dans les grès et les quartzites ne portent que quelques buissons épars à Bourdaine (Rhamnus frangula L.).

$X I I I-g 8$ : Les terroirs de «secano » et XIII-g9 : Les prairies de fauche des vallées alluviales, cf. XVII-g3 et XVII-g4.

par un pavage schisteux; litière éparse. - 3) l'érusion mécanique se limite au glissement du pavage et à quelques incisions des manteaux de débris. 


\section{Le géosystème continental "acide" du Bas Prioro (G. XVII).}

Les paysages austères du Bas Prioro annoncent les páramos de Castille tout en conservant un certain nombre de traits montagnards : un relief de collines convexes et de replats aux rebords disséqués qui a été taillé dans une masse schisteuse relativement homogène; un climat «continentalisé » de bassin, sec et lumineux, avec des inversions thermiques en hiver; une grande pauvreté floristique : les plantes des milieux humides ou subhumides (Hêtre, Chêne Sessile, Houx) ne dépassent pas les basses pentes des massifs périphériques alors que les plantes spécifiques des plateaux de Vieille Castille ne franchissent pas les gorges du Cea (c'est le cas de la Lavande pédonculée, du Ciste Ladanum, du Chêne de Lusitanie ...). C'cst, cnfin, lc secteur le plus cultivé et aussi le plus boisé (bien que le peuplement forestier y soit particulièrement médiocre). Les géofaciès forment des blocs compacts de grande taille de l'ordre du $\mathrm{km}^{2}$.

XVII-g1 : Les collines boisées à Chêne Tauzin couvrent près de la moitié de la surface du géosystème (23). Le sol est couvert en toutes saisons et il n'y a pas trace d'érosion mécanique. Par contre, ce géofaciès se caractérise par la surexploitation de sa couverture végétale : rotation trop rapide des coupes forestières, pacage abusif, incendies mal contrôlés. La biomasse est ainsi maintenue à un niveau très bas. Comme pour les autres terrains communaux du Valle de Prioro, le facteur écologique limitant est d'ordre juridico-économique (droits d'usage effectifs, absence d'aménagement forestier).

XVII-g2 : Les landes-pelouses xérophiles de versant ne se différencient de celles du géosystème XIII que par leur composition floristique : elles comportent une plus grande abondance d'espèces de milieux secs et dégradés (Genêt cendré, Calycotome épineux, Centaurées, Thyms, Hellébore, etc.).

XVII-g3 : Les terroirs de «secano» sont stables et rigoureusement délimités. Ils s'organisent en quartiers compacts sur les glacis inférieurs et sur certains hauts rcplats entre 1100 et $1400 \mathrm{~m}$ d'altitude. Le parcellaire, exigu et irrégulier, utilise en la remodelant la configuration du terrain (terrasses, rideaux, haies vives). Le système año y vez fait alterner, d'une sole à l'autre, cultures de céréales et jachères (ou du moins cultures légumières dérobées). Les friches sont rares. Les rendements en blé ne dépassant pas $10 \mathrm{q} / \mathrm{ha}$.

(23) Relevé-type : station à $1230 \mathrm{~m}$ d'altitude à la base d'un versant convexe, pente de $12 \%$, exposition SE. 1) taillis-fourré (4): Strate arborée avec Chêne Tauzin, $15 \overline{\mathrm{m}}$ de haut, branchu, âge $30-50$ ans; fourré (5): Aubépine (2), Genêt cendré (1), Callune (1), Héllébore fétide $(+)$, graminées (3). - 2) sol brun épaissi sur colluvion de bas de pente $(40-50 \mathrm{~cm})$
XVII-g4: Les prairies de fauche des vallées alluviales sont soigneusement entretenues et irriguées. Spongieuses et parfois noyées par les nappes phréatiques au moment des crues de fusion nivale, elles accusent en été un net déficit hydrique. Les sols présentent un horizon basal à pseudo-gley.

XVII-g5 : Les talus décapés à ravines et à cônes (commandement de 30 à $80 \mathrm{~m}$ ) sont les seuls géofaciès du Bas Prioro dont la structure et la dynamique soient directement liées à la géomorphogénèse. La partie supérieure convexe du talus est colonisée par une maigre lande à Callune et Bruyères (recouvrement : 20-30\%) sur des Rankers instables. Le ruissellement diffus s'organise progressivement en petites ravines qui s'anastomosent et alimentent, au bas des pentes, des pieds de versants de 2 à $4 \mathrm{~m}$ de hauteur sur 4 à $10 \mathrm{~m}$ de large. Ces dépôts meubles, mal fixés par la végétation (Fougeraie, Bruyères, buissons de Chêne Tauzin) sont disséqués et façonnés en petits cônes d'érosion par les ruissellements postérieurs. Les modelés de talus, d'échelle métrique ou décamétrique, sont actuels et anthropiques.

\section{Les “ cañadas".}

Les cañadas qui sillonnent le Valle de Prioro ne se surimposent pas à la mosaïque des géosystèmes. Elles sont les axes de dégradation à partir desquels progressent les formes d'érosion et de destruction du tapis végétal qui commandent l'évolution d'un certain nombre de géofaciès. Le tronc principal, balafre continue de 10 à $30 \mathrm{~m}$ de large, traverse du nord au sud le Valle de Prioro par les crêtes et la vallée centrale du Cea. C'est la voie de transhumance classique. Bien antérieure à la Mesta, elle fonctionne au moins depuis le Haut Moyen Age. Les cañadas secondaires se greffent sur cet axe ou divergent à partir des villages. Elles se perdent ensuite dans les pâturages en multiples digitations. Il aurait été plus logique de distinguer, dans chaque géosystème, un géofaciès de cañada. Mais il a paru plus important de saisir le phénomène dans son ensemble pour en examiner la signification écologique globale (fig. 4).

Les cañadas forment des milieux linéaires toujours plus dégradés et plus secs que les paysages environnants. Dans la bande centrale, le piétinement du bétail détruit la végétation, le sol et s'accompagne généralement de ravinements qui incisent la roche en place. Les roubines apparaissent chaque fois qu'il y a concentration des animaux (passage d'un gué, ou d'un col, périphérie des villages) ou chaque fois que la cañada recoupe un milieu fragile : talus entre deux glacis, soulane, dépôts meubles, talweg). Sur les marges de la cañada, le couvert végétal subsiste mais il est modifié par le pacage intensif, le tassement des sols et l'accumulation des déjections animales. Le cortège des plantes spécialisées est dominé par les Marrubes (Marrubium vulgare L.), le Carlin (Carduum carlinoïdes Gouan), les Centaurées (C. solsti- 
cialis L. et C. paniculata L.) qui s'accrochent à la toison des moutons, la Petite Oseille (Rumex aceto. sella L.) et quelques nitrophiles (Orties, etc.).

N'est-il pas possible, à titre d'hypothèse, de généraliser les observations réalisées le long des cañadas? Dans l'ensemble, la vie pastorale modifie les milieux dans le sens d'un assèchement édaphique (destruction des sols et des nappes phréatiques) et microclimatique (disparition ou éclaircissement du tapis végétal). Elle favorise donc l'implantation de plantes héliophiles et xérophiles qui n'appartiennent pas toutes au stock floristique local. Certaines peuvent être directement transportées par les troupeaux, d'autres peuvent s'installer d'elles-mêmes dans les stations dégradées et asséchées. On assisterait donc à une remontée, le long des cañadas, de certaines plantes ou même de certains cortèges floristiques. C'est évident pour le groupement végétal très spécialisé des Marrubes et des Chardons. Nous l'admettrons aussi pour le groupement végétal à Santoline, Thyms, Germandrées, etc., qui caractérise le géofaciès des soulanes à garide du géosystème montagnard calcaire (G. XX-g4, cf. p. 24 et p. 13). Cette explication n'estelle pas en fait valable pour tout le Valle de Prioro? Ne s'applique-t-elle pas aussi, d'une façon plus générale, à l'ensemble du versant sud cantabrique qui a été pendant des siècles soumis au système pastoral de la Mesta?

\section{LA DYNAMIQUE ACTUELLE DES PAYSAGES}

La dynamique «actuelle», c'est l'évolution des paysages à l'échelle humaine : l'unité de temps en est le siècle, voire la décennie. Elle s'inscrit dans le mouvement à plus large longueur d'onde du Quaternaire tout en conservant sa spécificité liée à l'emprise humaine. Dans cet espace-temps «historique», les milieux géographiques comme ceux du Valle de Prioro évoluent essentiellement sous l'effet d'un «système d'évolution anthropique» : assèchement édaphique et microclimatique, destruction ou édification de biomasses, géomorphogenèses épidermiques ou remodelages de versants, etc. Il faut remarquer que même dans le cas de ce système de mise en valeur sylvo-agro-pastoral de type traditionnel, peu mécanisé et motorisé, le rythme des transformations anthropiques est bien supérieur au rythme de reconstitution des structures naturelles. Le climax est rompu, du moins à l'échelle séculaire. Le cycle climacique, tout comme le cycle géomorphologique de W.M. Davis et des néo-davisiens, n'est qu'une dangereuse vue de l'esprit.

La mosaïque changeante des paysages s'inscrit dans l'espace et dans le temps. L'application d'une taxonomie à base «dynamique » permet de définir les ensembles géographiques du Valle de Prioro les uns par rapport aux autres (4 et 14).
A l'échelle des géofaciès, les situations «dynamiques» sont très différenciées. On distingue :

- les géofaciès en biostasie subclimacique (les structures naturelles sont peu altérées, l'unité est relativement stable). Ex. : XX-g1;

- les géofaciès en biostasie paraclimacique (stabilisation sur des structures nouvelles après une phase de régression peu marquée). Ex. : XX-g2-g3, XIII-g1, XIII-g3-g4, XVII-g1);

- les géofaciès en régression sur le plan des structures biotiques mais sans modification sensible du potentiel abiotique. Ex. : XVII-g2;

- les géofaciès en régression où la destruction des structures biotiques s'accompagne d'un certain nombre de modifications du potentiel abiotique. Ex. : XX-g6, XIII-g5-g6;

- les géofaciès en rhexistasie (géomorphogenèse dominante). Ex. : XX-g-4-g5, XVII-g5, les «cañadas»; - les géofaciès de substitution. Ex. : XX-g7, XIIIg2, XVII-g3-g4.

Les géofaciès de dégradation (régression biotique, abiotique et rhexistasie) l'emportent très largement sur les géofaciès en biostasie : ils couvrent près de $80 \%$ de la surface totale de Valle de Prioro, soit environ $40 \mathrm{~km}^{2}$.

A l'échelle des géosystèmes, cette dégradation généralisée, quoique très diverse au niveau des géofaciès, s'organise sous deux aspects opposés :

- Le géosystème montagnard «calcaire » est relativement stable sur le plan spatial. Certes, il est un peu grignoté, vers le haut, par le géosystème supraforestier (du fait de la progression de la lande subalpine sur la Hêtraie) et, vers le bas, par le géosystème semi-montagnard «acide» (par l'intermédiaire de XX-g7). Il doit sa permanence d'ensemble au substratum calcaire qui bloque la combinaison écologique au niveau pédogénétique, floristique et hydrologique. Les affleurements calcaires constituent l'une des discontinuités majeures de l'espace du Valle de Prioro et, de ce fait, une des structures permanentes du paysage.

La dynamique interne du géosystème a un double effet : d'une part, l'extension des géofaciès les plus dégradés; d'autre part, l'accentuation des contrastes entre les géofaciès, par exemple entre les ombrées (XX-g1) et les soulanes (XX-g5).

- Par contre, les trois autres géosystèmes (G XIII, G XV, G XVII), qui s'étendent sur un même substratum édaphique, se dégradent en s'uniformisant. Des structures climaciques différenciées sont remplacées par un complexe de régression monotone et banal formé par la lande haute, la Fougeraie ou le taillis de Chêne Sessile-Tauzin en association avec des pavages mobiles ou des Rankers. Il s'agit là d'un exemple de «télescopage» des géosystèmes et de l'élaboration d'une nouvelle unité isomorphe entièrement dominée par la mise en valeur socio-économique.

Les contrastes écologiques qui existent aussi bien à l'échelle des géofaciès qu'à celle des géosystèmes 
correspondent donc à des situations dynamiques très différentes. On retrouve en la précisant la notion d' « hétérostasie» de H. Erhart (24).

- La dynamique des paysages du Valle de Prioro est de plus en plus liée aux rapides transformations du système de mise en valeur économique : augmentation de l'exode rural, motorisation et transformations agro-pastorales, développement d'une politique de reforestation et d'aménagement hydraulique.

\section{BIBLIOGRAPHIE}

\section{METHODOLOGIE}

[1] G. Bertrand, Esquisse biogéographique de la Liébana (Massif Cantabrique, Espagne). La dynamique actuelle des paysages. Rev. géogr. Pyrénées S.-O., 1964, p. 225-262.

(24) H. Erhart, Le genèse des sols en tant que phénomène géologique. Esquisse d'une théorie géologique et géochimique. Biostasie et rhexistasie. Paris, Masson, Evolution des sciences, $2^{*}$ édition 1967.
[2] G. Bertrand, Paysage et géographie physique globale. Esquisse méthodologique. Rev. géogr. Pyrénées S.-O., 1968, p. 249-272.

[3] G. Bertrand, Ecologie de l'espace géographique. C.R. Soc. Biogéographie, séance du $18.12 .69, n^{\circ} 406$, p. 195205.

\section{VALLE DE PRIORO}

[4] Cartes topographiques: Mapa militar itinerario de España 1/200 000, no 14; Mapa de España 1/50 000, Riaño $\mathrm{n}^{\circ} 105$.

[5] Photographies aériennes : Servicio geografico del ejercito. Riaño $n^{\circ} 105, n^{\circ} 43193$ à 43197 et $n^{\circ} 43306$ à 43311 .

[6] G. Bertrand, Morphostructures cantabriques. Rev. géogr. Pyrénées S.-O., 1971, p. 49-70 (une carte et une planche de coupes au 1/200000).

[7] J. MounIER, Carte des régimes thermo-pluviométriques dans l'Ouest et le Nord-Ouest de la Péninsule Ibérique $(1 / 1000000)$ avec notice explicative de $4 \mathrm{p}$., 1969. C.N.R.S., Recherches cartographiques appliquée au climat et à l'hydrologie, Equipe de recherche associée à la Faculté des Sciences de Grenoble. 